\title{
Deposition of Magnetic Carbon Nano Tubes with Chromium Seed
}

\author{
Ana P. Mousinho, Ronaldo D. Mansano, Nelson Ordonez \\ Polytechnic School, Electronic System Engineering, Universityof São Paulo, São Paulo, Brazil \\ Email:mansano@lsi.usp.br
}

How to cite this paper: Mousinho, A.P., Mansano, R.D. and Ordonez, N. (2020) Deposition of Magnetic Carbon Nano Tubes with Chromium Seed. World Journal of Nano Science and Engineering, 10, 27-35.

https://doi.org/10.4236/wjnse.2020.102003

Received: June 4, 2020

Accepted: June 27, 2020

Published: June 30, 2020

Copyright (c) 2020 by author(s) and Scientific Research Publishing Inc. This work is licensed under the Creative Commons Attribution International License (CC BY 4.0).

http://creativecommons.org/licenses/by/4.0/

\begin{abstract}
In this work, was obtained metallic decorated, single wall Carbon Nanotubes (SWCNTs) using High Density Chemical Vapor Deposition (HDPCVD) system on chromium thin films on a silicon wafers substrate. The characteristics of this deposition method are capacity of the segregation of metallic nanoparticlesas seed for the SWCNT growing. Use of magnetic particle decorated carbon nanotubes increases the applications in magnetic devices, magnetic memory, and magnetic oriented drug delivery. The CNTs' spectra show a unique emission band, but due to the presence of the chromium, the spectra obtained in this work showed many bands that are related to the CNTs with different diameters. The CNTs obtained by the HDPCVD system are highly aligned and showed metallic features. Results of this work proved the possibility of obtaining the controlled deposition of aligned single-walled CNTs forest films decorated with chromium and suggested future studies in magnetic devices applications.
\end{abstract}

\section{Keywords}

CNTs Forest, High Density Plasma Deposition, High-Aligned CNTs, Nanomaterials

\section{Introduction}

Carbon nanotubes (CNTs) were discovered in 1991 [1]. CNTs are a new form of carbon that shows an equivalent form a bi-dimensional grapheme sheet wrapped as a tube. Single-walled carbon nanotubes, made of cylindrically rolled grapheme sheets, have attracted a lot of attention due to their interesting and potentially useful electrical and mechanical properties. In particular, regarding their mechanical properties, they have been hailed as the flexible springs of nature. Single-walled carbon nanotubes can be conductive and semiconducting. The elec- 
trical properties of the carbon nanotubes depend on the diameter of the tubes and the chiral angle [2]-[7]. The CNTs exhibit excellent properties for many applications, including: electrical, electro-optical, optical, optoelectronic, mechanical, electromechanical, magnetic, electromagnetic, chemical, electrochemical, thermal and thermoelectric properties [8]. Additionally, their incredible strength (a consequence of the famously strong carbon bond in the tubular structure) poses further advantages in nano-mechanic applications. The outstanding properties of CNTs have established the CNT forest as a new class of optically transparent and electrically conductive material that can be used in a numerous application such as field emission displays, and transparent electrodes for optoelectronic devices. Successful implementation of CNTs forest for these applications requires high quality of deposition processes. Generally, carbon nanotubes are produced by three main techniques, arc discharge, laser ablation and chemical vapor deposition [7].

This work reported methods for obtaining high-aligned single-walled carbon nanotubes forest decorating with chromium at room temperature. For this was used high-density plasma chemical vapor deposition processes. The parameters of plasma processes were varied. Metal nanoparticles decorated single-walled CNTs have been extensively studied and have shown to cause great enhancement in sensing performance [8]. Due to the presence of chromium, the CNTs showed metallic properties. Scanning Electron Microscopy, Atomic Force Microscopy (AFM), micro-Raman Spectroscopy and X-Ray Diffraction (XRD) [9] [10] [11] [12] characterized the single-walled CNTs forest decorated with chromium. In general, the carbon nanotubes spectra show a unique emission band, but due to the presence of the chromium, the spectra showed many bands that are related with the carbon nanotubes with different diameters. The CNTs obtained in this work, can be used as photonic material, due to the unique structural and electrical properties. With these results it is possible to obtain controlled deposition of single-walled CNTs decorated with chromium by high-density plasma chemical vapor deposition system for many applications, in special for ferromagnetic applications, including applications in magnetic devices, magnetic memory, and magnetic oriented drug delivery [13] [14]. The main novelty of this work is the presentation of a segregation of chromium seed during the deposition and use of refractory metal for nano structuration of carbon thin films. The use of chromium possibility is to obtain a low oxidation magnetic nanoparticle.

\section{Materials and Methods}

\subsection{High-Density Plasma Chemical Vapor Deposition System}

In this work, carbon nanotubes were deposited in a High Density Plasma Chemical Vapor Deposition System [15]. In this case, was couple a planar coil to an RF system for plasma generation, and an electrostatic shield for plasma densification are used. In this mode, high-density plasmas are obtained. For the ion acce- 
leration, use another RF system in planar configuration (as seen in Figure 1).

The lower pressure that the system can reach is $2 \times 10^{-7}$ Torr. In the HDPCVD system, there are two gas distributors. The main distributor was localized in the cover of the chamber, where a small homogenization chamber (secondary distributor of gases) exists. A holed ring made the distribution of the gases in the process chamber. The HDPCVD system has been created to work with gases (six different types) and liquids (two different types) at the same time. Thus, it is possible to create carbon nanotubes with additives (nitrogen, fluorine, oxygen, etc.).

\subsection{Samples, Deposition Processes and Characterization}

For the growth of the SWCNT forest, pure methane plasma processes are were used. Silicon wafer as substrate is used [15], for chromium (Cr) deposition using a Magnetron Sputtering System (250 W, 5 mTorr, 20-sccm argon for $15 \mathrm{~min}$ $90^{\circ} \mathrm{C}$ temperature and a chromium target) as the precursor material on the substrates. The carbon nanotubes were grown after the sample preparation (cleaning in piranha solution followed for HF dip) with the parameters: 15 mTorr, 250 $\mathrm{W}$ (coil power, RF, $13.56 \mathrm{MHz}$, remote plasma), 40-sccm methane and the deposition time was varied (1, 2, 3 and 4 hours), ambient temperature (Table 1). Raman Spectroscopy is used to characterize the structuration and the hybridization of the single-walled carbon nanotubes forest decorated with chromium. For the characterization of the SWCNTs structure was used an atomic force microscope (AFM), scanning electron microscope (SEM) and XRD Rigaku diffractometer (goniometer with $1^{\circ} / \mathrm{min}$ of speed, cupper $\operatorname{RX}$ tube $(\lambda=1.542 \AA$ )).

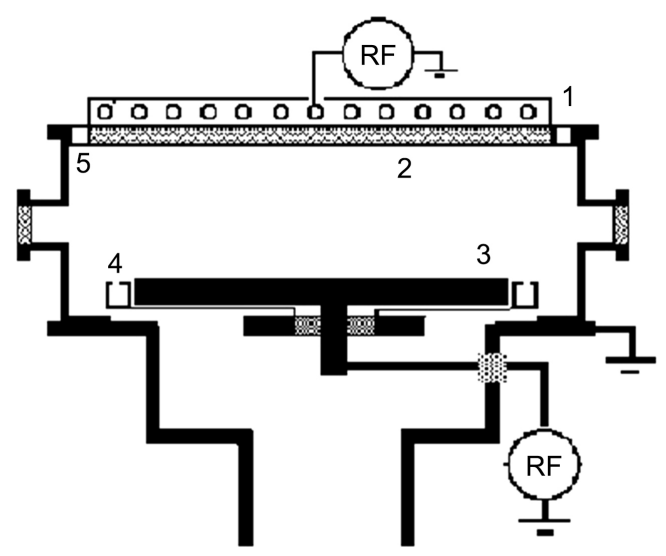

Figure 1. Schematically drawing of HDPCVD system. (1) Planar coil, (2) Dielectric window, (3) Electrode, (4) Secondary distributor of gases, and (5) Main distributor of gases.

Table 1. Deposition parameters of chromium thin film and CNT.

\begin{tabular}{cccccc}
\hline Material & Pressure & RF Power & Deposition Time & Deposition Technic & Gas \\
\hline Cr & 5 mTorr & $250 \mathrm{~W}$ & $15 \mathrm{~min}$ & Sputtering & Argon \\
CNT & 5 mTorr & $250 \mathrm{~W}$ & $1 \mathrm{~h}, 2 \mathrm{~h}, 3 \mathrm{~h}, 4 \mathrm{~h}$ & HDPCVD & CH4 \\
\hline
\end{tabular}




\section{Results and Discussion}

Using the Raman Spectroscopy technique it is possible to determine the structural properties, $(n, m)$ index, chirality, etc., but sometimes it is necessary to use a complementary techniques, because when use chemical vapour deposition with methane gas for growing the SWCNTs, complex dissociations occur that could create a large variety of structural characteristics. In this work, obtained high-aligned SWCNT forest decorated with chromium, and a high-density plasma chemical vapor deposition technique for deposition processes. These results can be observed in the micro Raman spectra. A typical spectrum for a unique carbon nanotube presents four characteristics vibrational modes: RBM, G band, D band and G' band. In this work, note the presence of these characteristics vibrational modes in CNTs forest films decorated with chromium and some new vibrational modes related to carbon nanotubes with different diameters when use the HDPCVD system. A typical micro-Raman spectrum obtained for carbon nanotubes decorated with chromium obtained in this work is showed in Figure 2.

The vibrational modes in CNTs forest films decorated with chromium are centred in $\sim 180 \mathrm{~cm}^{-1}, \sim 210 \mathrm{~cm}^{-1}, \sim 330 \mathrm{~cm}^{-1}$ and $\sim 460 \mathrm{~cm}^{-1}$. These peaks are related to the Radial Breathing Mode (RBM) and the presence of carbon nanotubes in the samples. The combination of the peakcentered in $\sim 180 \mathrm{~cm}^{-1}$ and the G band centered in $1560-1600 \mathrm{~cm}^{-1}$ are related with the strong presence of armchair SWCNTs in the samples. The vibrational modes centered in $\sim 210$ $\mathrm{cm}^{-1}, \sim 330 \mathrm{~cm}^{-1}$ and $\sim 460 \mathrm{~cm}^{-1}$ are peaks of the second order (Table 2). They are related with CNTs with different diameters. The presence of many vibrational modes indicates that obtain SWCNTs with a large range of the diameters due the presence of the chromium in the samples. The intensity of the peaks in the micro-Raman spectra increases when increase the final time deposition process.

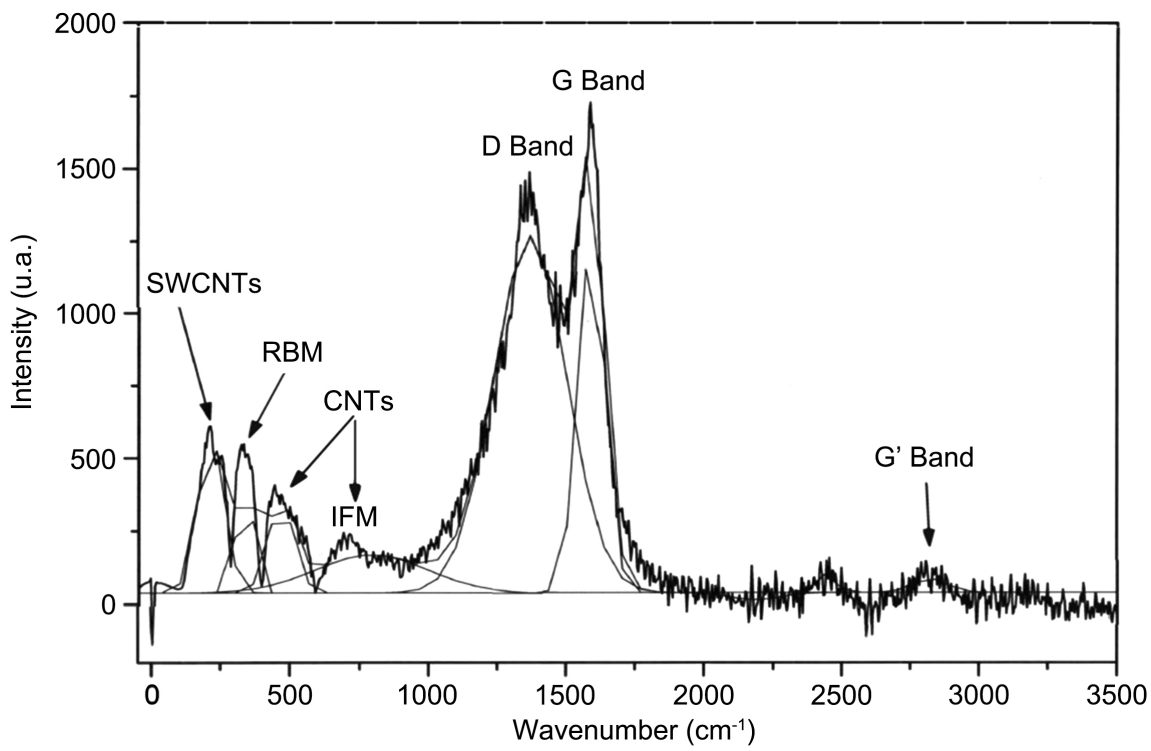

Figure 2. Typical micro-Raman spectrum for CNTs obtained in this work. 
When increase the deposition time, the intensities of the micro-Raman peaks increase. This effect occurs due more conformity of the nano-structure formation during the growth of the CNTs due more deposition time.

Figure 3 shows a typical x-ray diffraction pattern for CNTs obtained by HDPCVD system decorated with chromium. As expected for nanomaterials obtained spectra are noisy and difficult to interpret so for the determination of the position of the diffraction peaks curve fitting was performed, what enable to explain shape of the noisy XRD patterns correctly. The combination of linear and Gaussian functions was used for modeling XRD patterns. The diffraction peaks in XRD pattern at $5^{\circ}, 8^{\circ}$ and $28^{\circ}$ can be assigned to reflections from the (100) and (002) planes for carbon nanotubes and the pattern at $41^{\circ}$ can be assigned to reflections from the (200) plane for chromium. The diffraction peaks in XRD pattern obtained for CNTs decorated with chromium indicating a well-definition of the peaks and the presence of CNTs with high-alignment. These results prove the results obtained with the micro-Raman) spectroscopy technique (Table 3 ).

Table 2. Raman peaks of SWCNT forest.

\begin{tabular}{cc}
\hline Bonding & Peaks \\
\hline RBM & $180 \mathrm{~cm}^{-1}, 210 \mathrm{~cm}^{-1}, 330 \mathrm{~cm}^{-1}, 460 \mathrm{~cm}^{-1}$ \\
D band & $1330 \mathrm{~cm}^{-1}$ \\
G band & $1560-1600 \mathrm{~cm}^{-1}$ \\
G' band & $2700 \mathrm{~cm}^{-1}$ \\
\hline
\end{tabular}

Table 3. XRD peaks position of chromium thin film and CNT.

\begin{tabular}{ccc}
\hline Technic of analyses & Peaks CNT & Peak Cr \\
\hline XRD & $5^{\circ}, 8^{\circ}, 28^{\circ}$ & $41^{\circ}$ \\
\hline
\end{tabular}



Figure 3. Typical XRD patterns obtained for CNTs obtained in this work. 
The AFM images are showed in Figure 4. SEM micrographs in Figures 5-7 show the effect of the final deposition time in the CNTs forest growth. Figure 8 shows the fractured surface of CNT nanotubes forest after 3 hours of deposition time, in this image was possible observation of the nanotube formation.

Observing the AFM image and the SEM micrographs is can observe that the carbon nanotubes grow up in "clusters" (like a bouquet of flowers). These clusters have high-density of CNTs due the presence of chromium in the samples. The presence of chromium generates a roughness surfaces in the silicon wafers and promotes locally an increase of carbon-carbon bonds nucleation, indicating the carbon films nano-structuration and the high-aligned carbon nanotubes deposition. The total deposition time influences the carbon films structure formation and the carbon nanotubes formation, as can observed in the previous figures. The CNTs clusters formation regions occur due the chromium presence. The CNTs clusters formation regions occur due the chromium presence.

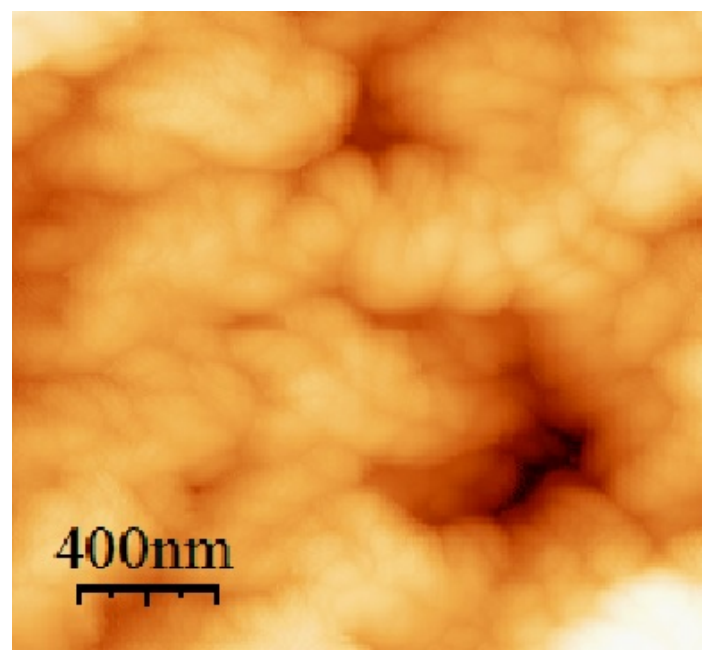

Figure 4. AFM image for SWCNTs forest decorated with chromium (deposition time of 4 hours).

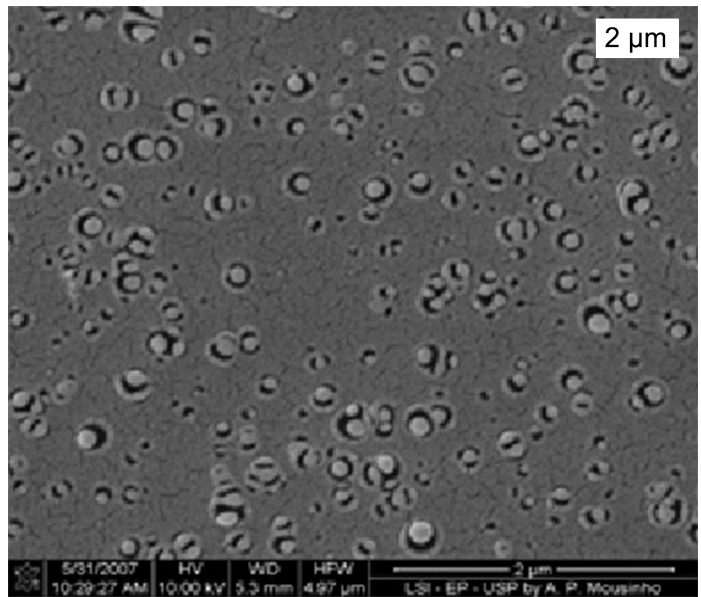

Figure 5. SEM micrograph for SWCNTs after 1 hour. deposition time. 


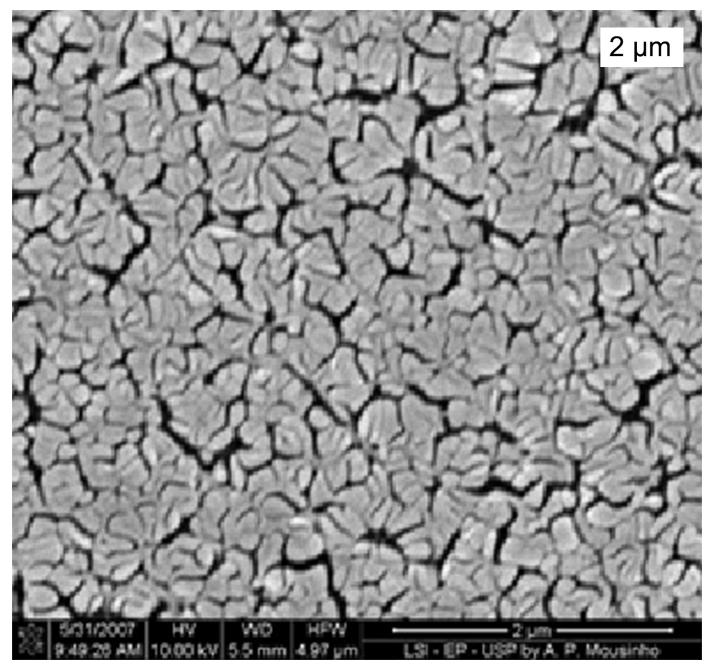

Figure 6. SEM micrograph for SWCNTs after 2 hours deposition time.

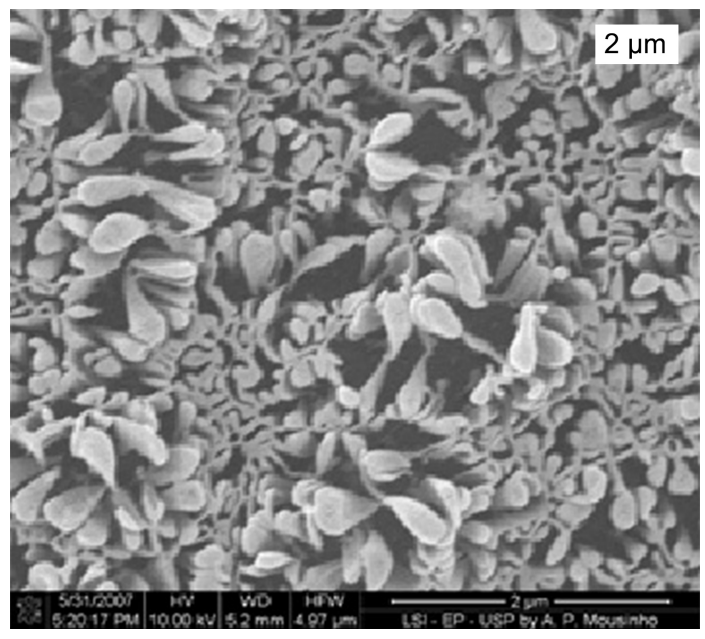

Figure 7. SEM micrograph for SWCNTs after 4 hours of deposition time.

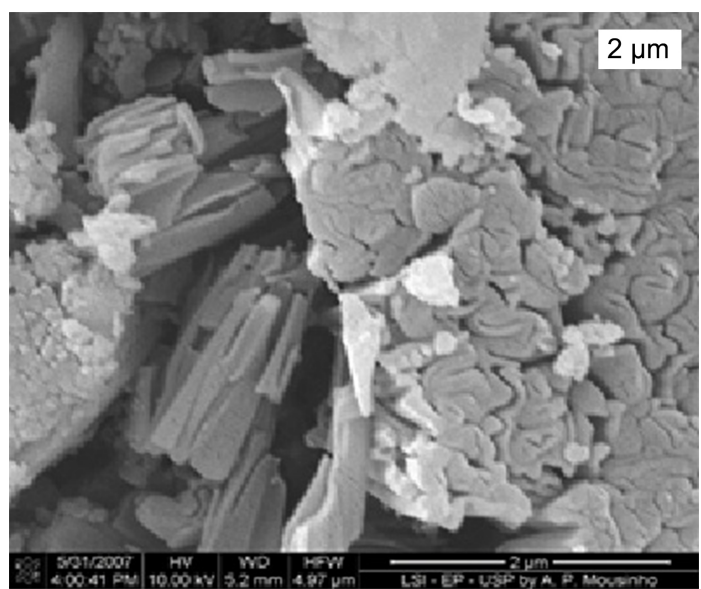

Figure 8. SEM micrograph of fractured surface of SWCNTs after 3 hours deposition time. 
With the SEM micrographs waspossible observe the stages of the CNTs growth. In the first stages the roughness of the films is low, so the CNTs growth is very faint. When increase the final deposition time, it was possible notice the cluster formation of CNTs forest (Figure 8). Due the internal mechanical stress, CNTs forest obtained with long final deposition time can break. The preferential growth of the CNTs in the samples occurs due the differences in the superficial energy in the samples, promoted by the chromium presence. The best process for obtaining SWCNTS by HDPCVD system is with 4 hours of process deposition at longer times the effect of films conformity (CNTs forest films with low superficial roughness. The AFM image showed in this work, prove this effect.

\section{Conclusion}

In this work, was obtained high-aligned single-walled carbon nanotubes forest decorated with chromium by high-density plasma chemical vapor deposition system at room temperature. The nano-material was analyzed by micro-Raman spectroscopy, XRD, SEM and AFM microscopy. The CNTs obtained in this work showed unique structural properties. These results shown the possibility obtaining single-walled carbon nanotubes decorated with chromium for many applications, in special for ferromagnetic applications and SWCNT-based electronic and optoelectronic devices.

\section{Acknowledgements}

The authors gratefully acknowledge the Dr. Adir José Moreira and Mr. Alexandre Camponucci, for providing technical support, and FAPESP and $\mathrm{CNPq}$ for financial support.

\section{Conflicts of Interest}

The authors declare no conflicts of interest regarding the publication of this paper.

\section{References}

[1] Fisher, J.F. (2006) Chapter 4 Carbon Nanotubes: Structure and Properties. In: Nanomaterials, CRC Press \& Francis, Boca Raton, 80-116.

[2] Asyraf, M.R.M. (2018) Optical Characterization of Tip Bended Vertically Aligned Carbon Nanotubes Array. Chemical Physics Letters, 711, 37. https://doi.org/10.1016/j.cplett.2018.09.011

[3] Kramberger, C., Pfeiffer, R., Kuzmany, H., Zólyomi, V. and Kürti, J. (2003) Assignment of Chiral Vectors in Carbon Nanotubes. Physical Review B, 68, Article ID: 235404. https://doi.org/10.1103/PhysRevB.68.235404

[4] Antunes, E.F., Lobo, A.O. and Trava-Airoldi, V.J. (2007) Influence of Diameter in the Raman Spectra of Aligned Multi-Walled Carbon Nanotubes. Carbon, 45, 912-921. https://doi.org/10.1016/j.carbon.2007.01.003

[5] Thomsen, C. and Reich, S. (2018) Raman Scattering in Carbon Nanotubes. Proceedings of SPIE - The International Society for Optical Engineering, 108, 115-234. https://doi.org/10.1007/978-3-540-34436-0 3 
[6] Zhang, W.D., et al. (2003) Vertically Aligned Carbon Nanotubes Grown in Different Types of Surface. Journal of Diamond and Related Materials, 12, 2175-2177. https://doi.org/10.1016/S0925-9635(03)00255-3

[7] Vivekchand, S.R.C. and Govindaraj, A. (2003) A New Method of Preparing Single-Walled Carbon Nanotubes. Proceedings of the Indian Academy of Sciences: Chemical Sciences, 115, 509-518. https://doi.org/10.1007/BF02708242

[8] Mubeen, S., Lim, J.H., Srirangarajan, A., Mulchandani, A., Deshusses, M.A. and Myung, N.V. (2011) Gas Sensing Mechanical Decorated Single-Walled Carbon Nanotubes. Electroanalysis, 23, 2687-2692. https://doi.org/10.1002/elan.201100299

[9] Doorn, S.K. (2006) Raman Spectroscopy of Single-Walled Carbon Nanotubes: Probing Electronic and Chemical Behavior. In: Carbon Nanotubes, Science and Applications, CRC Press, Boca Raton, Cap. 6, 154-176. https://doi.org/10.1201/9781315222127

[10] Stoner, B.R., Brown, B. and Glass, J.T. (2014) Selected Topics on the Synthesis, Properties, and Applications of Multiwalled Carbon Nanotubes. Journal of Diamond and Related Materials, 42, 49-57. https://doi.org/10.1016/j.diamond.2013.12.003

[11] Li, S., et al. (2012) In Situ Characterization of Structural Changes and the Fraction of Aligned Carbon Nanotube Networks Produced by Stretching. Carbon, 50, 3859-3867. https://doi.org/10.1016/j.carbon.2012.04.029

[12] Belin, T. and Epron, F. (2005) Characterization Methods of Carbon Nanotubes: A Review. Materials Science and Engineering. B, 119, 105-118. https://doi.org/10.1016/j.mseb.2005.02.046

[13] Samadishadlou, M., Farshbaf, N., Annabi, et al. (2018) Magnetic Carbon Nanotubes: Preparation, Physical Properties, and Applications in Biomedicine. Journal of Artificial Cells, Nanomedicine and Biotechnology, 46, 1314-1330. https://doi.org/10.1080/21691401.2017.1389746

[14] Anzar, N., Hasan, R., Tyagi, M., Yadav, N. and Narang, J. (2020) Carbon Nanotubes-A Review on Synthesis, Properties and Plethora of Applications in the Field of Biomedical Science. Sensor International, 1, Article ID: 100003. https://doi.org/10.1016/j.sintl.2020.100003

[15] Mansano, R.D. and Mousinho, A.P. (2011) Electro-Optical Properties of Carbon Nanotubes Obtained by High Density Plasma Chemical Vapor Deposition. Materials Sciences and Applications, 2, 381-389. https://doi.org/10.4236/msa.2011.25049 